\title{
Photodissociation Dynamics of the tert-Butyl Perthiyl Radical
}

\author{
Bethan Nichols, ${ }^{\mathrm{a}, *}$ Erin N. Sullivan* and Daniel M. Neumark ${ }^{1}$ \\ Department of Chemistry, University of California, Berkeley, California 94720, USA \\ and Chemical Sciences Division, Lawrence Berkeley National Laboratory, Berkeley, California \\ 94720, USA
}

\begin{abstract}
The photodissociation dynamics of the tert-butyl perthiyl ( $t$-BuSS) radical are investigated by fast-beam coincidence translational spectroscopy. A fast $(6-8 \mathrm{keV})$ beam of neutral $t$-BuSS radicals is produced via photodetachment of the corresponding anion, followed by photodissociation at $248 \mathrm{~nm}(5.00 \mathrm{eV})$ or $193 \mathrm{~nm}(6.42 \mathrm{eV})$ and coincident detection of the neutral products. Photofragment mass and translational energy distributions are obtained at both wavelengths. At $248 \mathrm{~nm}$, the dominant product channel (90\%) is found to be S loss, with a product translational energy distribution that peaks close to the maximum available energy and an anisotropic photofragment angular distribution, indicating dissociation along a repulsive excited state. A minor channel (10\%) leading to the formation of $\mathrm{S}_{2}+t$-Bu is also observed. At $193 \mathrm{~nm}$, both two- and three-body dissociation are observed. Formation of $\mathrm{S}_{2}+t$-Bu is the dominant two-body product channel, with multiple electronic states of the $S_{2}$ molecule produced via excited state dissociation processes. Formation of $\mathrm{S}+t$-BuS is a minor two-body channel at this dissociation energy. The three-body channels are $\mathrm{S}_{2}+\mathrm{H}+$ isobutene, $\mathrm{S}_{2}+\mathrm{CH}_{3}+$ propene, and $\mathrm{S}+\mathrm{SH}+$ isobutene. The first two of these channels result from a sequential dissociation process in which loss of $\mathrm{S}_{2}$ from $t$-BuSS results in ground state $t$-Bu with sufficient internal energy to undergo secondary fragmentation. The third three-body channel, $\mathrm{S}+\mathrm{SH}+$ isobutene, is attributed to loss of internally excited $\mathrm{HS}_{2}$ from $t$-BuSS, which then rapidly dissociates to form $\mathrm{S}+\mathrm{SH}$ in an asynchronous concerted dissociation process.
\end{abstract}

\footnotetext{
${ }^{1}$ Corresponding author. Email: dneumark@berkeley.edu

${ }^{a}$ Present Address: Thumbtack, San Francisco, CA 94103, USA

*These authors contributed equally to this work.
} 


\section{Introduction}

The disulfide bonding motif is found in a variety of arenas throughout chemistry ranging from chemical biology, where it is critical in the folding and stability of proteins, ${ }^{1,2}$ to the vulcanization of rubber in industry, where disulfide bonds are used to cross link between different polymer chains. ${ }^{3}$ In mammalian tissue, high levels of hydropersulfides (RSSH) have been detected ${ }^{4}$ and recent studies have proposed that these persulfides play an important role as radical trapping antioxidants, leading to the formation of the corresponding perthiyl radicals (RSS), which can then further react, fragment, or dimerize. ${ }^{5,6}$ Despite the potential importance of these perthiyl radicals as intermediates in disulfur chemistry, comparatively little work has been done to characterize their spectroscopy and photochemistry other than methyl perthiyl $\left(\mathrm{CH}_{3} \mathrm{SS}\right)$, which has been previously studied in our group and elsewhere. ${ }^{7-9}$ In this article, we investigate the photodissociation of the tert-butyl perthiyl radical (t-BuSS) at $248 \mathrm{~nm}(5.00 \mathrm{eV})$ and $193 \mathrm{~nm}$ $(6.42 \mathrm{eV})$ in order to determine its primary photochemistry and dissociation mechanisms.

There have been very few experimental or theoretical investigations of the spectroscopy of $t$ BuSS. Early experiments characterized $t$-BuSS radicals produced from photolysis of $t$-BuSSCl using electron spin resonance spectroscopy. ${ }^{10}$ Additionally, photolysis of tert-butyl tetrasulfide has been used to produce $t$-BuSS radicals, which exhibit a strong absorption in the UV around $375 \mathrm{~nm}(3.31 \mathrm{eV}) .{ }^{11,12} \mathrm{~A}$ theoretical study of the structures of the simplest perthiyl radicals and their anions predicted a value of $1.847 \mathrm{eV}$ for the electron affinity of the $t$-BuSS radical. ${ }^{13}$ Calculations performed in this work and presented in the supplementary material indicate the ground state is of ${ }^{2} A "$ symmetry

The spectroscopy and photodissociation dynamics of the related methyl perthiyl radical have received significantly more attention. Moran and Ellison ${ }^{7}$ used anion photoelectron spectroscopy to determine the electron affinity of $\mathrm{CH}_{3} \mathrm{SS}(1.757 \mathrm{eV})$ and vibrational frequencies of the $\mathrm{S}-\mathrm{S}$ stretching mode for the anion and neutral. The photodissociation dynamics of $\mathrm{CH}_{3} \mathrm{SS}$ radicals have also recently been studied at $248 \mathrm{~nm}$ and $193 \mathrm{~nm} .{ }^{8,9}$ At $248 \mathrm{~nm}$, the dominant product channel is $\mathrm{S}+\mathrm{CH}_{3} \mathrm{~S}$ formed via repulsive dissociation on an electronically excited state, with a small contribution from $\mathrm{S}_{2}+\mathrm{CH}_{3}$ products. At $193 \mathrm{~nm}, \mathrm{~S}$ loss remains the major dissociation 
channel, although $\mathrm{S}_{2}$ loss becomes more competitive, accounting for around one third of the fragmentation products. Both $\mathrm{S}$ and $\mathrm{S}_{2}$ products are produced in multiple electronic states, suggesting excited state dissociation processes. CASSCF/CASPT2 calculations ${ }^{14}$ predict that the first two excited states of $\mathrm{CH}_{3} \mathrm{SS}$ lie $1.05 \mathrm{eV}$ and $3.42 \mathrm{eV}$ above the ground state respectively, and so are not likely to contribute to the absorption at $248 \mathrm{~nm}(5.00 \mathrm{eV})$ and $193 \mathrm{~nm}(6.42 \mathrm{eV})$. Calculations of higher lying electronic states have yet to be performed.

Perthiyl radicals are the sulfur analog of peroxy radicals, which are key intermediates in hydrocarbon oxidation. ${ }^{15}$ Recent fast radical beam studies in our laboratory investigated photodissociation dynamics of methyl, ethyl and $t$-butyl peroxy radicals at $248 \mathrm{~nm} .{ }^{16,17}$ The alkyl peroxy radicals were excited to their $\widetilde{B}$-state and coincident detection of the photofragments yielded information on the two- and three-body dissociation channels. In these species, the $\tilde{B}$ state is repulsive along the $\mathrm{O}-\mathrm{O}$ bond stretching coordinate, and its photoexcitation results in loss of $\mathrm{O}$ atoms on electronically excited states, leading to both two- and three-fragment dissociation channels. As the alkyl substituent increases in size, internal conversion to the ground electronic state becomes increasingly competitive, leading to loss of $\mathrm{O}_{2}$ and $\mathrm{HO}_{2}$ fragments. It is of interest to compare the alkyl perthiyl and alkyl peroxy photodissociation dynamics to explore differences in the photochemistry of the sulfur and oxygen analogues.

In this work, we carry out fast radical beam experiments to investigate the photodissociation dynamics of the $t$-butyl perthiyl ( $t$-BuSS) radical at $248 \mathrm{~nm}(5.00 \mathrm{eV})$ and $193 \mathrm{~nm}(6.42 \mathrm{eV})$. Multiple two- and three-body dissociation channels are energetically accessible at these energies: ${ }^{18-20}$

$$
\begin{aligned}
\mathrm{C}_{4} \mathrm{H}_{9} \mathrm{SS} & \rightarrow \mathrm{S}+\mathrm{C}_{4} \mathrm{H}_{9} \mathrm{~S}(t-\mathrm{BuS}) & \mathrm{D}_{0}=3.48 \mathrm{eV} \\
& \rightarrow \mathrm{S}_{2}+\mathrm{C}_{4} \mathrm{H}_{9}(t-\mathrm{Bu}) & \mathrm{D}_{0}=2.04 \mathrm{eV} \\
& \rightarrow \mathrm{SH}+\mathrm{C}_{4} \mathrm{H}_{8} \mathrm{~S} \text { (dimethylthirane) } & \mathrm{D}_{0}=1.77 \mathrm{eV} \\
& \rightarrow \mathrm{HS}_{2}+\mathrm{C}_{4} \mathrm{H}_{8} \text { (isobutene) } & \mathrm{D}_{0}=1.04 \mathrm{eV} \\
& \rightarrow \mathrm{S}_{2}+\mathrm{H}+\mathrm{C}_{4} \mathrm{H}_{8} \text { (isobutene) } & \mathrm{D}_{0}=3.62 \mathrm{eV} \\
& \rightarrow \mathrm{S}_{2}+\mathrm{CH}_{3}+\mathrm{C}_{3} \mathrm{H}_{6} \text { (propene) } & \mathrm{D}_{0}=3.27 \mathrm{eV} \\
& \rightarrow \mathrm{S}_{2}+\mathrm{CH}_{3}+\mathrm{CH}_{3} \mathrm{CCH}_{3} \text { (dimethyl carbene) } & \mathrm{D}_{0}=5.86 \mathrm{eV}
\end{aligned}
$$




$$
\begin{array}{ll}
\rightarrow \mathrm{S}+\mathrm{SH}+\mathrm{C}_{4} \mathrm{H}_{8} \text { (isobutene) } & \mathrm{D}_{0}=4.35 \mathrm{eV} \\
\rightarrow \mathrm{S}+\mathrm{CH}_{3}+\mathrm{C}_{3} \mathrm{H}_{6} \mathrm{~S} \text { (thioacetone) } & \mathrm{D}_{0}=5.03 \mathrm{eV}
\end{array}
$$

Photodissociation of $t$-BuSS at $248 \mathrm{~nm}$ yields near exclusive formation of channel $1(\mathrm{~S}+t$-BuS), with a minor contribution from channel $2\left(\mathrm{~S}_{2}+t\right.$-Bu) products. At $193 \mathrm{~nm}$, channel 1 is minor and $\mathrm{S}_{2}$ loss becomes the major dissociation pathway, leading to the two-body dissociation products $\mathrm{S}_{2}+t$ - $\mathrm{Bu}$, as well as three-body product channels 5 and $6\left(\mathrm{~S}_{2}+\mathrm{H}+\right.$ isobutene and $\mathrm{S}_{2}+$ $\mathrm{CH}_{3}+$ propene, respectively) from the secondary dissociation of energized $t$-Bu fragments. Finally, the three-body dissociation products formed via channel $8(\mathrm{~S}+\mathrm{SH}+$ isobutene $)$ are observed and are attributed to the loss of internally excited $\mathrm{HS}_{2}$, followed by dissociation of the $\mathrm{HS}_{2}$ molecule.

\section{Experimental}

The fast-beam coincidence translational spectrometer employed in this study has been described in detail previously, ${ }^{8,21}$ so only the details specific to this work will be discussed here. While this instrument was originally designed to measure two-body photodissociation events, more recent detector configurations have enabled coincidence-based detection of three-body dissociation. $^{22,23}$

A fast beam of tert-butyl perthiyl anions $\left(t\right.$-BuSS$\left.{ }^{-}\right)$was generated by flowing $15 \mathrm{psig}$ (1 bar) $\mathrm{Ar}$ through di-tert-butyl disulfide. The gas mixture was supersonically expanded into the vacuum through an Amsterdam Piezovalve ${ }^{24}$ operating at $100 \mathrm{~Hz}$, coupled with a DC grid discharge source $^{25}$ to produce $t$-BuSS ${ }^{-}$ions. The ions were accelerated to a beam energy of $6-8 \mathrm{keV}$ and mass-selected using a Bakker-type mass spectrometer. ${ }^{26,27}$ Mass-selected $t$-BuSS ${ }^{-}$ions were subsequently photodetached with a $532 \mathrm{~nm}(2.33 \mathrm{eV})$ pulse from a Nd:YAG laser (Litron LPY742-100) or at $677 \mathrm{~nm}(1.83 \mathrm{eV})$ with a Nd:YAG-pumped dye laser (Radiant Dyes NarrowScan) to produce a fast beam of neutral $t$-BuSS.

The neutral $t$-BuSS radicals formed in the detachment step were characterized by a photoelectron spectrometer installed in the photodetachment region. ${ }^{8}$ The photodetached electrons were 
extracted perpendicular to the beam of the neutral radicals and velocity-mapped onto a positionsensitive detector consisting of a chevron stack of two multichannel plates (MCPs) and a phosphor screen. Events on the phosphor screen were captured by a camera and transferred to a computer for analysis of the resulting image. Photoelectron translational energy distributions were obtained from the recorded images by means of Abel inversion (BASEX), ${ }^{28}$ yielding information on the structure of the radical formed by photodetachment as well as the internal energy of the ions and neutrals.

After the photodetachment region, any remaining anions in the fast beam were deflected from the beam path using an electric field. The resulting beam of fast neutral $t$-BuSS was then intersected by a laser pulse from an excimer laser (GAM EX-50F) operating at either $248 \mathrm{~nm}$ $(5.00 \mathrm{eV})$ or $193 \mathrm{~nm}(6.42 \mathrm{eV})$. Photodissociation products that scatter from the beam path were detected in coincidence on a time-and-position-sensitive Roentdek Hex80 delay-line-anode detector, $23,29,30$ with any undissociated $t$-BuSS blocked by a $2.5 \mathrm{~mm}$ radius beam block in front of the detector face. For each coincident event, either a two-body or three-body dissociation, the arrival times and positions of the photofragments were determined and analyzed to yield the photofragment masses, translational energy release, and scattering angle. A satisfactory data set consists of greater than 10,000 coincident events, but an abundance of signal allowed for over 30,000 coincidence events for some of the results presented here.

The resulting two-body dissociation photofragment translational and angular distributions are given by the relation

$$
P\left(E_{T}, \theta\right)=P\left(E_{T}\right) \cdot\left[1+\beta\left(E_{T}\right) P_{2}(\cos \theta)\right]
$$

where $\beta\left(E_{T}\right)$ is the energy-dependent anisotropy parameter and $P_{2}$ is the 2 nd-order Legendre polynomial. ${ }^{31}$ The anisotropy parameter for three-body dissociation is calculated directly from the angular distributions of the scattered products. In the current experiments, the unpolarized output of the excimer laser is used for dissociation, so $\theta$ is defined as the angle between the dissociation recoil axis (or in the case of the three-body dissociation, the normal to the dissociation plane) and the direction of propagation of the laser. In the case of two-body 
dissociation, the observed value, $\beta_{\text {obs }}$, takes on values between -1 for parallel and $+1 / 2$ for perpendicular transitions, corresponding to the $\beta$ parameters for linearly polarized light $\left(\beta_{\text {lin }}\right)$ multiplied by $-1 / 2.8$

For events with very low or high kinetic energy release, or when there is a large fragment mass mismatch, one or more fragments can hit either the beam block or miss the detector entirely and therefore go undetected. In order to account for this variation of the detection efficiency as a function of scattering angle and kinetic energy release, the experimental translational energy distributions for two- and three-body dissociation events and corresponding Dalitz plots presented in this work have been corrected using a detector acceptance function (DAF).

\section{Results and Analysis}

\section{a. Photoelectron Spectrum}

Figure 1 shows the anion photoelectron spectrum for $t-\mathrm{BuSS}^{-}$at a detachment wavelength of 532 $\mathrm{nm}$. The main peaks in the photoelectron spectrum are labelled 1-10. Peak 1, located at an electron kinetic energy (eKE) of $0.524 \mathrm{eV}$, is assigned to the transition from the vibrational ground state of the anion to the vibrational ground state of the neutral radical $\left(0_{0}^{0}\right)$. For the detachment wavelength used in this study $(532 \mathrm{~nm}, 2.331 \mathrm{eV})$, this gives an electron affinity of the $t$-BuSS radical of $1.807(4) \mathrm{eV}$, in reasonable agreement with the value of $1.847 \mathrm{eV}$ from previous calculations at the Gaussian-3 level of theory. ${ }^{13}$ 


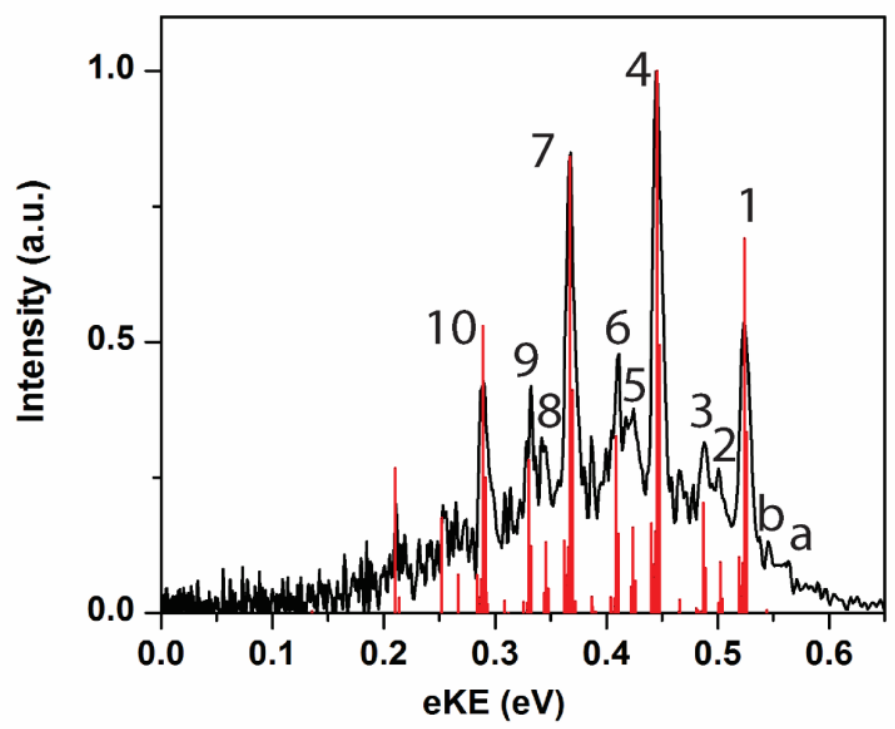

Figure 1. Experimental anion photoelectron spectrum of $t-\mathrm{BuSS}^{-}$at $\lambda=532 \mathrm{~nm}$ (black line), and Franck-Condon simulations at 200K (red).

To aid with assignment of the spectroscopic features labelled in the spectrum, Franck-Condon simulations were performed using the program ezSpectrum ${ }^{32}$ at an assumed ion temperature of $200 \mathrm{~K}$. Vibrational frequencies and normal coordinates for the anion and neutral species were calculated with density functional theory (DFT) using the B3LYP functional and the Dunningtype cc-pVDZ basis set augmented with diffuse functions using the Gaussian 09 package. ${ }^{33}$ Calculated neutral frequencies were scaled by a factor of 0.948 such that the $v_{16}$ fundamental (see below) matched experiment, and the origin of the simulated spectrum was shifted to the experimental band origin.

Franck-Condon simulations for the photodetachment of $t$-BuSS at a vibrational temperature of $200 \mathrm{~K}$ are shown as red lines in Figure 1 and give good agreement with the observed spectrum. One can then assign peaks 1-10 and a-b, as indicated in Table $\mathrm{S} 1$ in the supplementary material, and determine vibrational frequencies for several modes of the radical and anion. Error bars for the vibrational frequencies (given below) and electron affinity were obtained from the uncertainty in the peak position, given by the width parameter obtained from a Gaussian fit to the peak. 
Peak 4 is the largest peak in the spectrum, located at an eKE $0.078 \mathrm{eV}$ below the origin (Peak 1). This peak is part of the dominant progression in the spectrum, involving peaks 1, 4, 7 and 10 . This progression is assigned to the S-S stretching mode $\left(v_{16}\right)$ and the vibrational frequency of this mode is determined to be $v_{16}=632(33) \mathrm{cm}^{-1}$. Two additional lower frequency progressions can be observed: peaks 2, 5 and 8 , and peaks 3, 6, and 9. These are assigned to the $22_{0}^{1} 16_{0}^{n}$ and $21_{0}^{1} 16_{0}^{n}$ transitions, respectively, involving the low frequency modes $v_{21}$ and $v_{22}$. The $v_{22}$ mode corresponds to a C-S-S bending motion with a vibrational frequency of $186(33) \mathrm{cm}^{-1}$, and the $v_{21}$ mode, which is best described as symmetric $\mathrm{CH}_{3}$ internal rotations with some $\mathrm{C}-\mathrm{S}-\mathrm{S}$ bending character, has a frequency of $292(33) \mathrm{cm}^{-1}$. At eKEs slightly higher than the origin, there is evidence of two small peaks a and $\mathrm{b}$ that can be assigned to hot bands in the low frequency $v_{21}$ and $v_{22}$ modes.

The consistency between the experimental and Franck-Condon simulated photoelectron spectrum confirms the identity of the $t$-BuSS radical, and the distribution in Figure 1 reflects the range of internal energies of the $t$-BuSS radical produced by $532 \mathrm{~nm}$ photodetachment. The majority of the radicals are produced with up to $0.25 \mathrm{eV}$ of internal energy, localized in the $v_{16}$, $v_{21}$ and $v_{22}$, S-S stretching, $\mathrm{C}-\mathrm{S}-\mathrm{S}$ bending and $\mathrm{CH}_{3}$ torsional modes. For photodetachment at $677 \mathrm{~nm}$, only peak 1 is energetically accessible.

\section{b. Photofragment Mass Distributions}

Figure 2 shows the two-body photofragment mass distributions for dissociation of $t$-BuSS at 248 $\mathrm{nm}(5.00 \mathrm{eV})$ and $193 \mathrm{~nm}(6.42 \mathrm{eV})$. At $248 \mathrm{~nm}$, there is evidence for two dissociation channels: the dominant channel with peaks at $32 \mathrm{Da}$ and $89 \mathrm{Da}$, and a minor channel with mass peaks at 57 $\mathrm{Da}$ and $64 \mathrm{Da}$. The dominant channel could correspond to dissociation via channel $1(\mathrm{~S}+t$-BuS $)$ or channel 3 ( $\mathrm{SH}+$ dimethylthirane), both of which are energetically accessible. Contributions from these channels can overlap since our photofragment mass resolution is $m / \Delta m \sim 10 .{ }^{31,34}$ Figures S1 and S2 in the supplemental material present best fit simulations of the two-body distributions. The simulated distribution including channel 3 presents a slightly better match to the experimental distribution, but only predicts a small fraction of the distribution to be from 
channel 3. As we cannot further distinguish channel 1 and channel 3, we focus our analysis on channel 1 which is clearly the dominant channel. Likewise, the minor channel could correspond to formation of either $\mathrm{S}_{2}+t$-Bu (channel 2) or $\mathrm{HS}_{2}+$ isobutene (channel 4), but simulations of the two-body mass distribution support the formation of channel 2 only, with no contribution from $\mathrm{HS}_{2}$ loss as part of a two-body channel.

a)

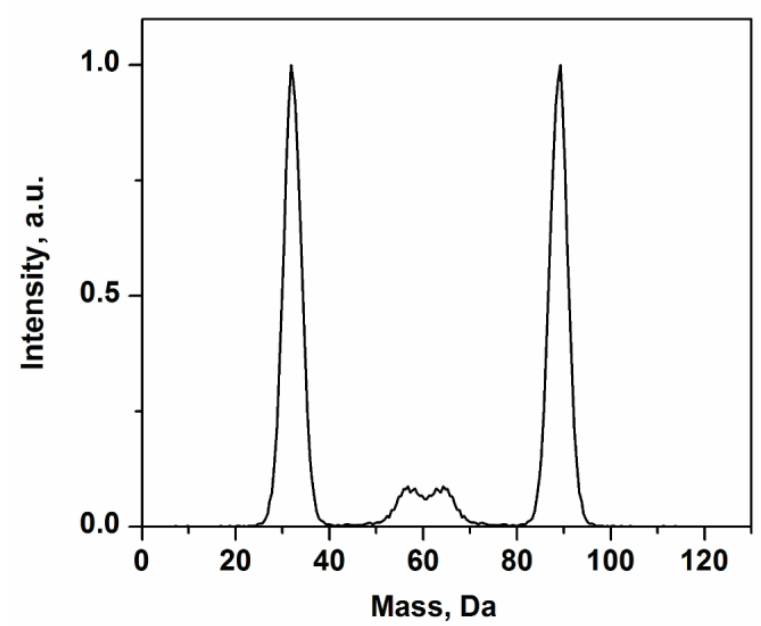

b)

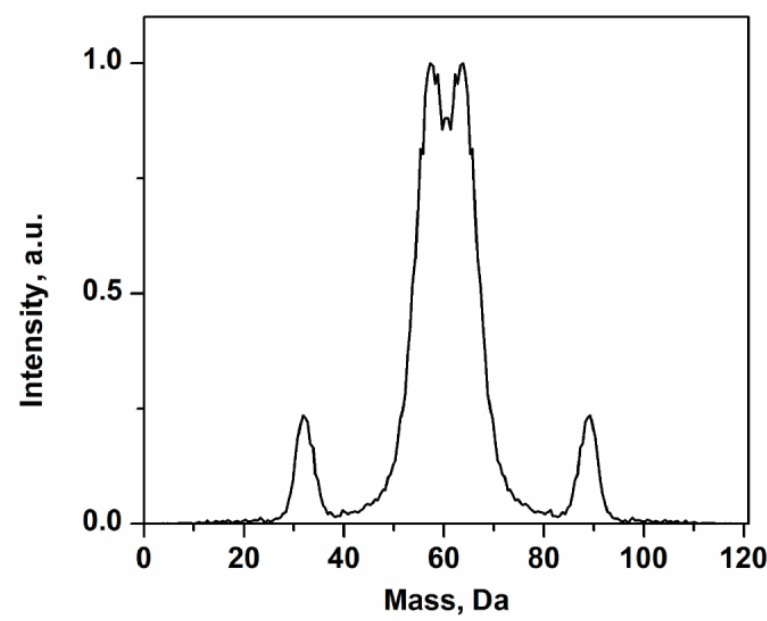

Figure 2. Photofragment mass distributions for two-body dissociation of $t$-BuSS at $248 \mathrm{~nm}$ (panel a) and $193 \mathrm{~nm}$ (panel b).

For dissociation at $193 \mathrm{~nm}$, the peak positions in the mass distribution remain unchanged, but the peaks at $57 \mathrm{Da}$ and $64 \mathrm{Da}$ are now much larger compared to those at $32 \mathrm{Da}$ and $89 \mathrm{Da}$. Again, simulations were performed to ascertain the contributions of product channels with similar masses and the best agreement with the experimental distribution was found for dissociation via channel 1 (minor channel) and channel 2 (dominant channel) with no evidence of contributions from channels 3 or 4. More detail regarding these simulations are presented in Figures S1 and S2 of the supplemental material.

Figure 3 shows the photofragment mass distribution for three-body dissociation of $t$-BuSS at 193 $\mathrm{nm}$. Two large peaks can be observed in the three-body mass distribution around $32.5 \mathrm{Da}$ and 56 $\mathrm{Da}$, consistent with the formation of $\mathrm{S}(32 \mathrm{Da})+\mathrm{SH}(33 \mathrm{Da})+$ isobutene $(56 \mathrm{Da})$ via channel 8. The peak at $32.5 \mathrm{Da}$ is roughly twice as intense as that at $56 \mathrm{Da}$ as the two individual mass peaks corresponding to the S and SH fragments cannot be resolved. 


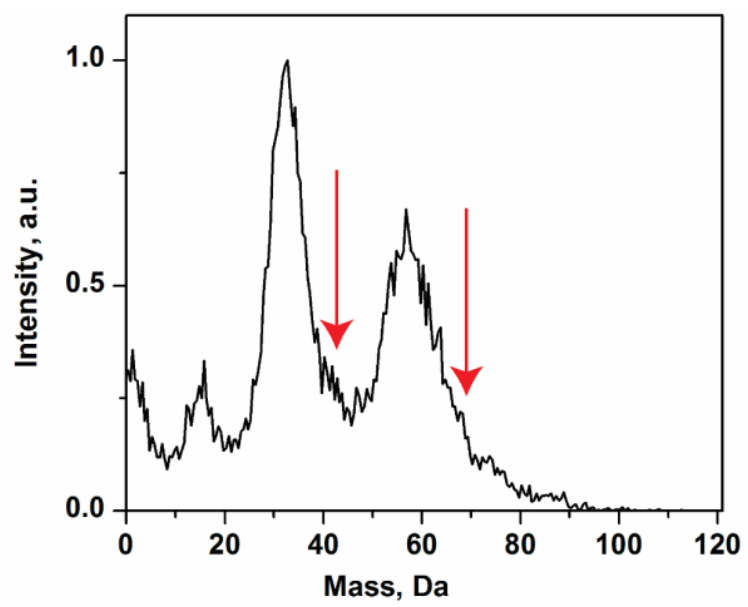

Figure 3. Photofragment mass distribution for three-body dissociation of $t$-BuSS at $193 \mathrm{~nm}$. Red arrows indicate shoulders to the main peaks due to the formation of $S_{2}$ and $\mathrm{C}_{3} \mathrm{H}_{6}$ of channel 6 or 7 with the remaining fragment corresponding to $\mathrm{CH}_{3}$ at $15 \mathrm{Da}$.

A small peak around $15 \mathrm{Da}$ is also clear in the three-body mass distribution, which could indicate formation of channel $6\left(\mathrm{~S}_{2}+\mathrm{CH}_{3}+\right.$ propene $)$, channel $7\left(\mathrm{~S}_{2}+\mathrm{CH}_{3}+\right.$ dimethylcarbene) or channel $9\left(\mathrm{~S}+\mathrm{CH}_{3}+\right.$ thioacetone), which all involve $\mathrm{CH}_{3}$ corresponding to mass $15 \mathrm{Da}$. The red arrows in Figure 3 highlight shoulders around $42 \mathrm{Da}$ and $64 \mathrm{Da}$ on the high mass side of the two main peaks and correspond to $\mathrm{C}_{3} \mathrm{H}_{6}$ and $\mathrm{S}_{2}$ via channel 6 or 7 . Channel 9 products would be analogous to the three-body dissociation channel observed in the photodissociation of $t$ - $\mathrm{BuOO}$ at $248 \mathrm{~nm}$, but the absence of significant intensity in the three-body mass distribution around $74 \mathrm{Da}$ (thioacetone) suggests that this is, at most, a very minor channel.

A further small peak is observed in the three-body mass distribution at around $1 \mathrm{Da}$, corresponding to the formation of channel $5\left(\mathrm{~S}_{2}+\mathrm{H}+\right.$ isobutene). It should be noted that due to the finite size of the detector and the presence of the beam block, dissociation events with large fragment mass ratios, such as production of $\mathrm{H}$ atoms and heavier products, are generally undetectable in coincidence and as such only a small fraction of the true coincident events will be detected in this experiment. 


\section{c. Photofragment Translational Energy Distributions and Dalitz Plots}

The translational energy available to the photofragments can be determined according to:

$$
E_{\mathrm{T}, \max }=h v+E_{\mathrm{int}}^{\mathrm{R}}-E_{\mathrm{int}}^{\mathrm{P}}-D_{0},
$$

where $h v$ is the photon energy, $E_{\mathrm{int}}^{\mathrm{R}}$ is the internal energy of the $t$-BuSS radicals prior to dissociation, $E_{\text {int }}^{\mathrm{P}}$ is the internal energy of the photofragments after dissociation, and $D_{0}$ is the bond dissociation energy. From the photoelectron spectrum presented in Section 3a, it is clear that for photodetachment at $532 \mathrm{~nm}(2.33 \mathrm{eV})$, the majority of the $t$-BuSS radicals are not produced in the vibrational ground state, but instead have an internal energy up to $\sim 0.25 \mathrm{eV}$, which should be considered when analyzing the experiments performed at this detachment wavelength. In order to assess the effect of vibrational excitation of the neutral $t$-BuSS prior to photodissociation, experiments were also performed in which the photodetachment wavelength was tuned to $677 \mathrm{~nm}(1.83 \mathrm{eV})$, very close to the electron affinity of $t$-BuSS $(1.81 \mathrm{eV})$; signal levels for these experiments were much lower due to the relatively poor Franck Condon overlap at the vibrational origin.

a)

b)
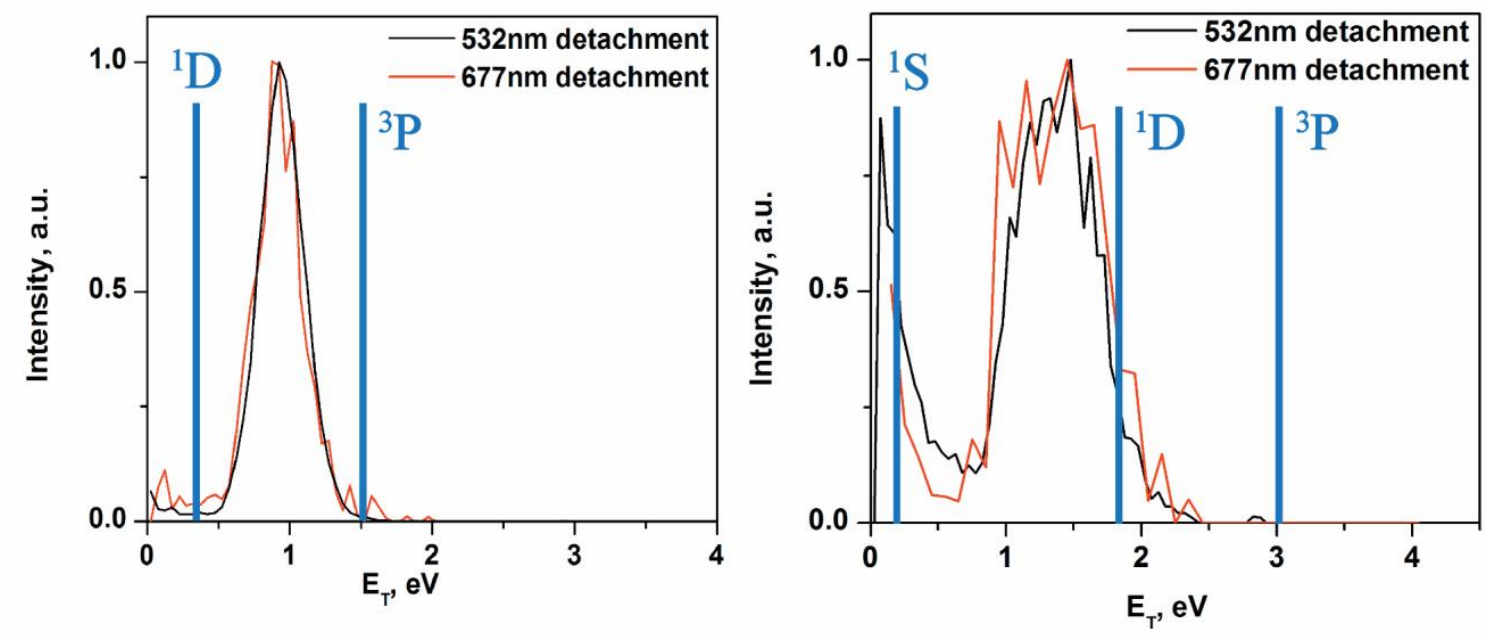

Figure 4. Photofragment translational energy distributions for dissociation of $t$-BuSS into $\mathrm{S}+t$-BuS at $248 \mathrm{~nm}$ (panel a) and $193 \mathrm{~nm}$ (panel b). $t$-BuSS radicals were formed via photodetachment of the corresponding anion at $532 \mathrm{~nm}$ (black) and $677 \mathrm{~nm}$ (red). The solid blue lines show the maximum available translational energy $\left(E_{\mathrm{T}, \mathrm{MAX}}\right)$ for the formation of $t$-BuS $+\mathrm{S}\left({ }^{3} \mathrm{P}\right), \mathrm{S}\left({ }^{1} \mathrm{D}\right)$ and $\mathrm{S}\left({ }^{1} \mathrm{~S}\right)$, assuming no internal excitation of the $t$ BuSS radicals prior to photodissociation. 
Figure 4 shows the photofragment translational energy distributions for the $\mathrm{S}+t$-BuS product mass channel at $248 \mathrm{~nm}$ and $193 \mathrm{~nm}$. At both wavelengths, multiple electronic states of the sulfur atom are electronically accessible, with $E_{\mathrm{T}, \mathrm{MAX}}$ for each channel as indicated in Figure 4. At 248 $\mathrm{nm}$, the translational energy distribution appears as a single narrow peak centered around $1.1 \mathrm{eV}$ and extending to approximately $1.8 \mathrm{eV}$, close to the maximum available energy $\left(E_{\mathrm{T}, \mathrm{MAX}}\right)$ for the $\mathrm{S}\left({ }^{3} \mathrm{P}\right)+t$-BuS channel. At this wavelength, both the $\mathrm{S}\left({ }^{3} \mathrm{P}\right)$ and $\mathrm{S}\left({ }^{1} \mathrm{D}\right)$ electronic states are energetically accessible. However, the peak in the translational energy distribution is above $E_{\mathrm{T}, \mathrm{MAX}}$ for the formation of $\mathrm{S}\left({ }^{1} \mathrm{D}\right)+t$-BuS, indicating that the $\mathrm{S}$ atom is formed only in the ${ }^{3} \mathrm{P}$ electronic state. There is little distinction in the translational energy release for experiments performed at different detachment wavelengths. Dissociation events for this channel appear anisotropic, with a measured $\beta$ parameter of $\beta_{\text {obs }}=-0.34 \pm 0.03$; this corresponds to $\beta_{\text {lin }}=$ 0.68 for linearly polarized light, indicating a propensity for the photofragments to recoil parallel to the direction of the electric field vector if a linearly polarized laser was used.

For dissociation to $\mathrm{S}+t$-BuS at $193 \mathrm{~nm}$, the photofragment yield of $\mathrm{S}$ loss is substantially lower than those results at $248 \mathrm{~nm}$, as is evident in the mass distributions in Fig. 2. Regardless, the photofragment translational energy distribution shows two distinct peaks, one centered at $1.5 \mathrm{eV}$ and extending to around $3.0 \mathrm{eV}$ and the other peaking close to $0.1 \mathrm{eV}$ and extending to $0.5 \mathrm{eV}$. $E_{\mathrm{T}, \mathrm{MAX}}$ for the formation of each of the $\mathrm{S}$ atom electronic states is shown by a solid line in Figure 4. Similar to dissociation at $248 \mathrm{~nm}$, the higher translational energy release peak is anisotropic with $\beta_{\text {obs }}=-0.40 \pm 0.06$, or $\beta_{\text {lin }}=0.80$, again corresponding to a parallel transition. 
a)

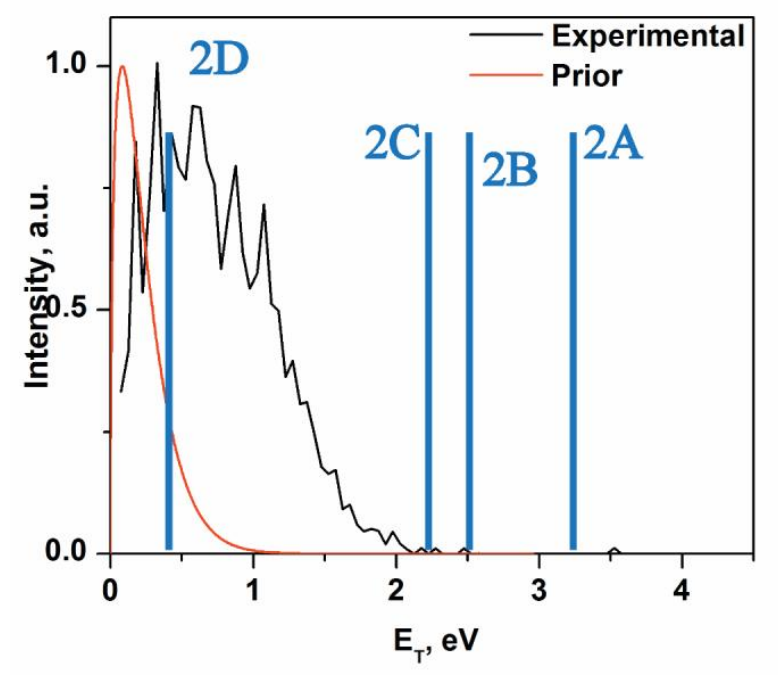

b)

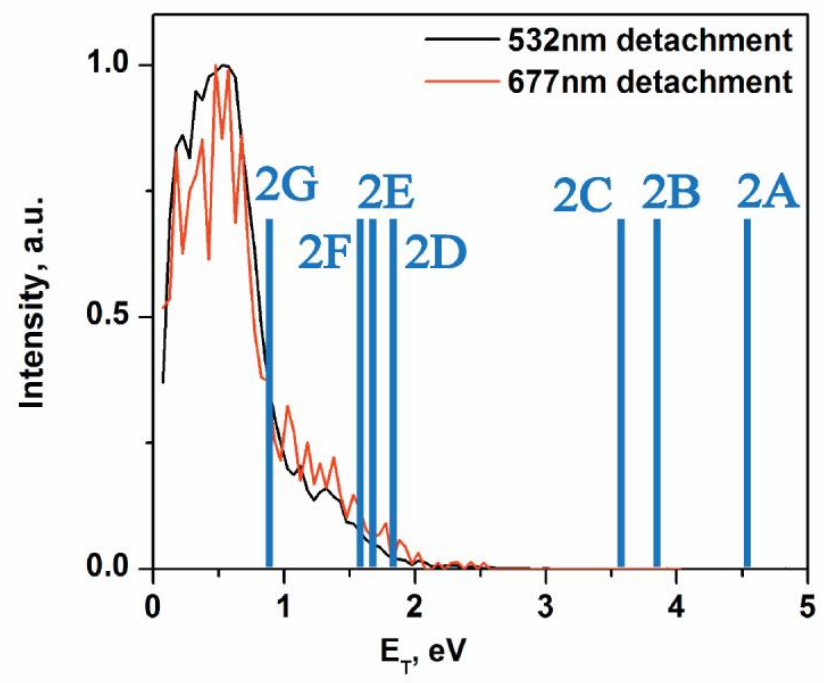

Figure 5: Photofragment translational energy distributions for dissociation of $t$-BuSS into $\mathrm{S}_{2}+t$ $\mathrm{Bu}$ at $248 \mathrm{~nm}$ (panel a) and $193 \mathrm{~nm}$ (panel b). At $248 \mathrm{~nm}$ the experimental distribution (black) is compared with a calculated prior distribution (red). At $193 \mathrm{~nm}$ the two experimental distributions obtained at different detachment wavelengths are shown in black $(532 \mathrm{~nm})$ and red $(677 \mathrm{~nm})$. The blue vertical lines show $E_{\mathrm{T}, \mathrm{MAX}}$ for the formation of $t-\mathrm{Bu}+\mathrm{S}_{2}$ in electronic states indicated in Table I.

The translational energy distributions for dissociation of $t$-BuSS to form $\mathrm{S}_{2}+t$-Bu at $248 \mathrm{~nm}$ and $193 \mathrm{~nm}$ are shown in Figure 5. At both wavelengths, multiple electronic states of the $\mathrm{S}_{2}$ fragments are accessible. The energetics for these processes are outlined in Table I with values obtained from both experimental and theoretical sources. ${ }^{20,35}$

Table I. Energetically accessible product channels for the $\mathrm{S}_{2}+t$-Bu mass channel at $248 \mathrm{~nm}$ and $193 \mathrm{~nm}$. All energies are in $\mathrm{eV} . E_{\mathrm{T}, \mathrm{MAx}}$ values include $0.25 \mathrm{eV}$ of internal energy in the initial $t$ BuSS radical.

\begin{tabular}{lllll}
\hline Product channel & $D_{0}$ & $E_{\mathrm{T}, M A X}$ at $248 \mathrm{~nm}$ & $E_{\mathrm{T}, M A X}$ at $193 \mathrm{~nm}$ & Label \\
\hline $\mathrm{S}_{2}\left(X^{3} \Sigma_{g}^{-}\right)+t-\mathrm{Bu}\left(\tilde{X}^{2} \mathrm{~A}_{1}\right)$ & 2.04 & 3.21 & 4.63 & $2 \mathrm{~A}$ \\
$\mathrm{~S}_{2}\left(a^{1} \Delta_{g}\right)+t-\mathrm{Bu}\left(\tilde{X}^{2} \mathrm{~A}_{1}\right)$ & 2.75 & 2.50 & 3.92 & $2 \mathrm{~B}$ \\
$\mathrm{~S}_{2}\left(b^{1} \Sigma_{g}^{+}\right)+t-\mathrm{Bu}\left(\tilde{X}^{2} \mathrm{~A}_{1}\right)$ & 3.03 & 2.22 & 3.64 & $2 \mathrm{C}$ \\
$\mathrm{S}_{2}\left(c^{1} \Sigma_{u}^{-}\right)+t-\mathrm{Bu}\left(\tilde{X}^{2} \mathrm{~A}_{1}\right)$ & 4.81 & 0.44 & 1.86 & $2 \mathrm{D}$ \\
$\mathrm{S}_{2}\left(A^{\prime 3} \Delta_{u}\right)+t-\mathrm{Bu}\left(\tilde{X}^{2} \mathrm{~A}_{1}\right)$ & 4.97 & 0.28 & 1.70 & $2 \mathrm{E}$ \\
$\mathrm{S}_{2}\left(A^{3} \Sigma_{u}^{+}\right)+t-\mathrm{Bu}\left(\tilde{X}^{2} \mathrm{~A}_{1}\right)$ & 5.07 & 0.18 & 1.60 & $2 \mathrm{~F}$ \\
$\mathrm{~S}_{2}\left(X^{3} \Sigma_{g}^{-}\right)+t-\mathrm{Bu}\left(\tilde{A}^{2} \mathrm{~A}_{1}\right)$ & 5.78 & - & 0.89 & $2 \mathrm{G}$ \\
\hline
\end{tabular}


At $248 \mathrm{~nm}$, the translational energy distribution peaks close to $0.4 \mathrm{eV}$ and extends to around 2.1 $\mathrm{eV}$, close to $E_{\mathrm{T}, \mathrm{MAX}}$ for product channels $2 \mathrm{~A}, 2 \mathrm{~B}$ and $2 \mathrm{C}$. The angular distributions for this channel are isotropic, with $\beta_{\mathrm{obs}}=-0.01 \pm 0.1$. For dissociation at $193 \mathrm{~nm}$, the translational energy distribution for the $\mathrm{S}_{2}+t$-Bu product mass channel peaks close to $0.7 \mathrm{eV}$, with a sharp drop in intensity around $1.0 \mathrm{eV}$, and a smaller shoulder that continues out to $2.0 \mathrm{eV}$, slightly above $E_{\mathrm{T}, \mathrm{MAX}}$ for the formation of product channels $2 \mathrm{D}-2 \mathrm{~F}$. This mass channel shows slightly anisotropic distributions of the photofragments with $\beta_{\mathrm{obs}}=-0.18 \pm 0.04$. 
a)

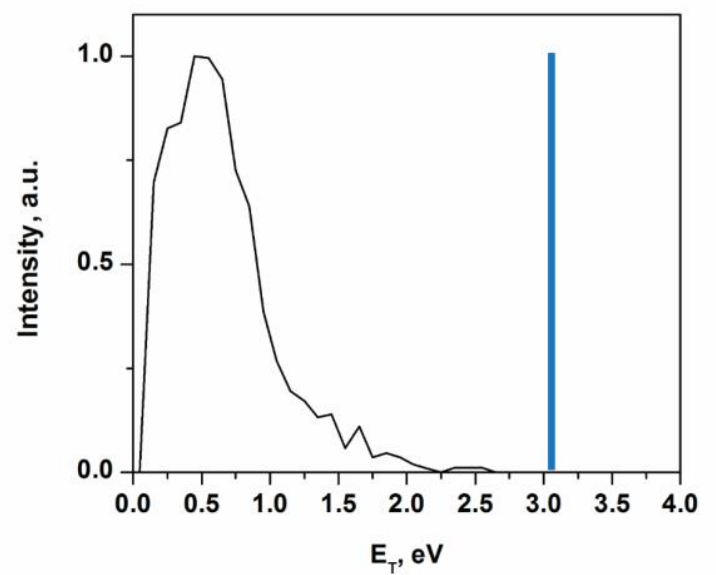

b)

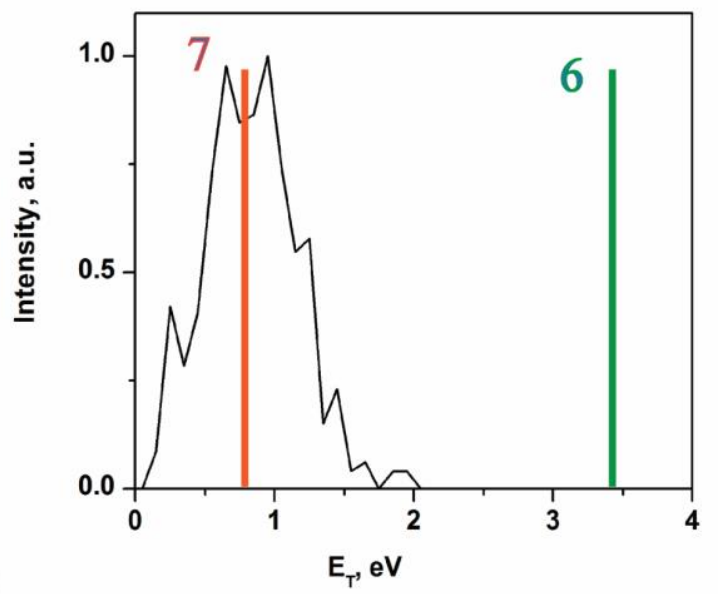

c)

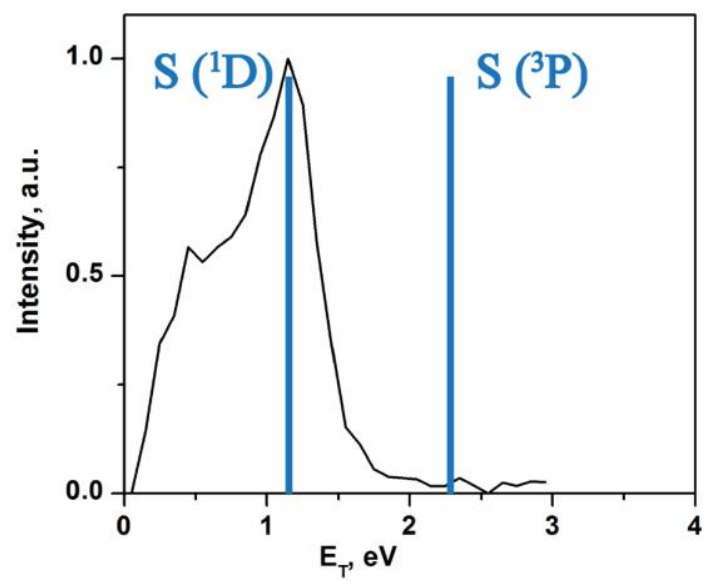

Figure 6. Photofragment translational energy distributions for three-body dissociation of t-BuSS to $\mathrm{S}_{2}+\mathrm{H}+$ isobutene (panel a), $\mathrm{S}_{2}+\mathrm{CH}_{3}+\mathrm{C}_{3} \mathrm{H}_{6}$ (panel b) and $\mathrm{S}+\mathrm{SH}+$ isobutene (panel c) at $193 \mathrm{~nm}$. The green and orange vertical lines in panel b) show $E_{\mathrm{T}, \mathrm{MAX}}$ formation of product channel $6\left(\mathrm{~S}_{2}+\mathrm{CH}_{3}+\right.$ propene $)$ and channel $7\left(\mathrm{~S}_{2}+\mathrm{CH}_{3}+\right.$ dimethyl carbene $)$, respectively. The blue vertical lines in panel c) show $E_{\mathrm{T}, \mathrm{MAX}}$ for formation of the sulfur atom in the ${ }^{3} \mathrm{P}$ and ${ }^{1} \mathrm{D}$ electronic states. The $E_{\mathrm{T}, \mathrm{MAX}}$ values include $\sim 0.25 \mathrm{eV}$ of internal energy in the initial $t$-BuSS radical. 
Translational energy distributions for the three-body product channels observed at $193 \mathrm{~nm}$ are shown in Figure 6. Panel a) displays the translational energy distribution for $\mathrm{S}_{2}+\mathrm{H}+$ isobutene (channel 5). The distribution peaks around $0.5 \mathrm{eV}$, with intensity observed up to around $2.5 \mathrm{eV}$. The angular distribution for channel 5 is isotropic, with $\beta_{\text {obs }}=-0.08 \pm 0.06$. Interestingly, the distribution in Fig. 6a looks almost identical to that in Fig. 5b. The similarity between the distributions in Figures $6 \mathrm{a}$ and $5 \mathrm{~b}$ is mostly likely an artifact of false coincidence events in which an erroneous particle is detected in addition to a two-body event generating channel 2 . This phenomenon will be addressed below.

The translational energy distribution for $\mathrm{S}_{2}+\mathrm{CH}_{3}+\mathrm{C}_{3} \mathrm{H}_{6}$ products, shown in Figure $6 \mathrm{~b}$, peaks around $0.7 \mathrm{eV}$, with intensity observed up to around $2.0 \mathrm{eV}$. Since the intensity in the translational energy distribution extends well beyond $E_{\mathrm{T}, \mathrm{MAX}}$ for the formation of product channel $7\left(\mathrm{~S}_{2}+\mathrm{CH}_{3}+\right.$ dimethyl carbene $)$, the products are attributed to channel $6\left(\mathrm{~S}_{2}+\mathrm{CH}_{3}+\right.$ propene $)$. The photofragment angular distribution is isotropic with $\beta_{\text {obs }}=-0.01 \pm 0.05$.

Figure $6 \mathrm{c}$ shows the photofragment translational energy distribution for channel $8(\mathrm{~S}+\mathrm{SH}+$ isobutene) at $193 \mathrm{~nm}$. The main peak in the distribution is centered at $1.2 \mathrm{eV}$ and extends to around $2.0 \mathrm{eV}$, close to $E_{\mathrm{T}, \mathrm{MAX}}$ for the formation $\mathrm{S}\left({ }^{3} \mathrm{P}\right)$. A shoulder at lower energy can be observed around $0.8 \mathrm{eV}$, close to $E_{\mathrm{T}, \mathrm{MAX}}$ for the production of $\mathrm{S}\left({ }^{1} \mathrm{D}\right)$. The angular distributions in both regions are anisotropic, with $\beta_{\text {obs }}=0.20 \pm 0.05$ for dissociation events above $0.8 \mathrm{eV}$ and $\beta_{\text {obs }}=0.13 \pm 0.06$ below $0.8 \mathrm{eV}$. 
a)

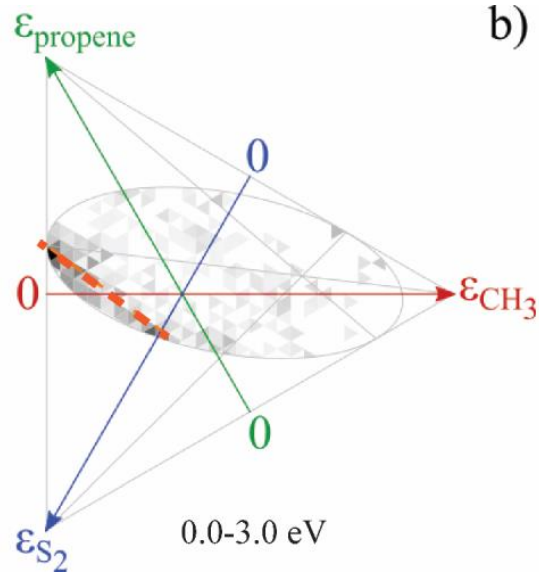

b)

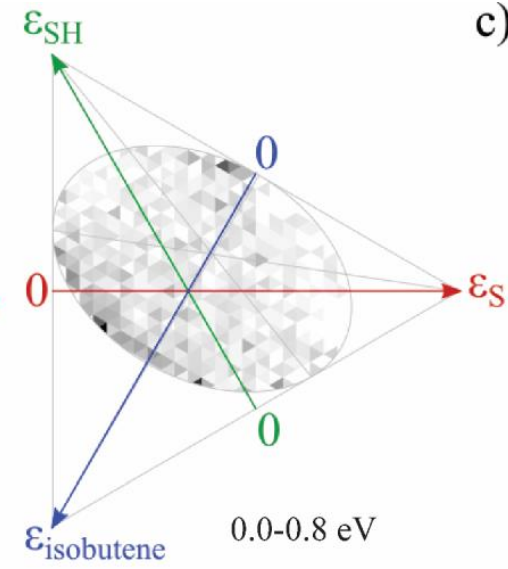

c)

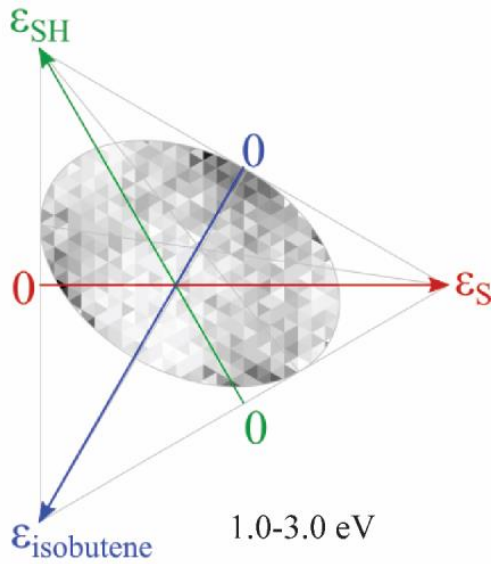

Figure 7. Panel a) Dalitz plot for the three-body dissociation channel $6, \mathrm{~S}_{2}+\mathrm{CH}_{3}+$ propene, showing the energy partitioning amongst the $\mathrm{CH}_{3}$ (red arrow), propene (green arrow) and $\mathrm{S}_{2}$ (blue arrow). Panels b) and c) Dalitz plots for channel 8 showing the energy partitioning amongst the $\mathrm{S}$ (red), SH(green) and isobutene (blue) photofragments, integrated over the translational energy ranges $0.0-0.8 \mathrm{eV}$ and $1.0-3.0 \mathrm{eV}$ respectively. Relative intensities are indicated by shades of grey ranging from white (zero intensity) to black (maximum intensity).

Further insight into the three-body dissociation dynamics are provided by Dalitz plots shown for the $\mathrm{CH}_{3}+\mathrm{S}_{2}+$ propene and $\mathrm{S}+\mathrm{SH}+$ isobutene channels in Figure 7.23,37,38 These plots show the translational energy partitioning between each photofragment $i$, with each of the fragments having an energy fraction (represented by the arrows in Figure 7) given by $\epsilon_{i}=E_{i} / E$ for each event with total translational energy $E$. Each point on the Dalitz plot provides information about the energy partitioning between the fragments. All dissociation events are restricted to lie within the triangle by conservation of energy, and within the inscribed ellipse by conservation of momentum. The Dalitz plot for channel $6\left(\mathrm{CH}_{3}+\mathrm{S}_{2}+\right.$ isobutene), shown in Figure $7 \mathrm{a}$, shows a stripe of intensity, highlighted by the orange dashed line, corresponding to fast $S_{2}$ fragments. The Dalitz plot for channel $5\left(\mathrm{~S}_{2}+\mathrm{H}+\right.$ isobutene) is not presented here; only $\mathrm{H}$ atoms with very low translational energies are detected in this experiment, so the Dalitz plot contains little useful information. It is shown in the supplementary material for completeness (Figure S2).

Dalitz plots for channel $8(\mathrm{~S}+\mathrm{SH}+$ isobutene) are shown in Figure 7 integrated over the shoulder (0-0.8 eV, panel b) and main peak (1.0-3.0 eV, panel c) of the translational energy distribution. The low translational energy Dalitz plot shows considerable shot noise due to the small number of events in this energy range. It should also be noted that due to the similar masses of the $\mathrm{S}$ and $\mathrm{SH}$ fragments, the analysis program may incorrectly label some $\mathrm{S}$ fragments 
as SH and vice versa. For low translational energy release events (between 0-0.8 eV), the Dalitz plot in Fig. $7 \mathrm{~b}$ shows the greatest intensity in the bottom left region of the ellipse, corresponding to fast isobutene fragments. At higher translational energy release, the region with the greatest intensity is found at the base of the blue arrow, corresponding to slow isobutene fragments.

\section{d. Product Branching Ratios}

The branching ratios for each channel are shown in Table II. The branching ratios are obtained from the raw experimental counts, assuming a one-particle detection efficiency of $p=0.6 .{ }^{23}$ The two-body channels are corrected using $p_{\text {two-body }}=0.36$, and the three-body channels are corrected using $p_{\text {three-body }}=0.22$. A further correction is made for channel 5 which involves $\mathrm{H}$ loss, as the probability of detecting an $\mathrm{H}$ atom is even smaller than 0.6 ; instead, $p_{\mathrm{H} \text {-atom }}=0.08$ so $p_{\text {channel } 5}=$ $0.03 .{ }^{39}$ At $248 \mathrm{~nm}$, the products are dominated by loss of a sulfur atom to form the $t$-BuS radical. Additionally, a small amount of $\mathrm{S}_{2}+t$-Bu radical formation is observed. At $193 \mathrm{~nm}$, the product branching ratios change substantially; $\mathrm{S}_{2}+t$-Bu production is the dominant dissociation channel, while $\mathrm{S}+t$-BuS accounts for only $7 \%$ of the products. Each branching ratio comes from averaging the results of each data set and thus, the errors associated with each channel are random error.

At $193 \mathrm{~nm}$, the similarity between the translational energy distributions in Figs. 5b and 6a leads us to suspect that the contribution from channel 5 (Fig. 6a) is exaggerated due to false coincidence events in which three fragments hit the detector that are from distinct two-body events. In this situation, two of the fragments have a physically viable center-of-mass, i.e. they are channel 2 fragments from the same dissociating $t$-BuSS radical. The most common assignment of the third fragment is hydrogen because it shifts the center of mass from its true value minimally, and thus, these events appear as channel 5. Such a mechanism would explain the similar translational energy distributions in Figs. 5b and 6a. This is not to say, however, that channel 5 is absent, but it is obscured by these false coincidences. One reason channel 5 is particularly susceptible to being overshadowed is the low probability of all three $\mathrm{S}_{2}+\mathrm{H}+$ isobutene fragments hitting our detector. For even a very small translational energy release $(<0.5$ $\mathrm{eV}$ ), the likelihood of all three fragments of a channel 5 event is only about $25 \%$, whereas it is 
about three times that for a channel not involving $\mathrm{H}$ loss (i.e. channel 6). Therefore, the $39 \%$ value for channel 5 presented in middle column of Table II is likely an overestimate.

At $193 \mathrm{~nm}$, the loss of $\mathrm{S}_{2}$ in its ground or first two low-lying states leaves the $t$-Bu radical with sufficient energy to dissociate into either $\mathrm{H}+\mathrm{C}_{4} \mathrm{H}_{8}$ or $\mathrm{CH}_{3}+$ propene, and $\mathrm{RRKM}$ calculations (Section 4) find the $\mathrm{H}: \mathrm{CH}_{3}$ branching ratio is about 6.4. The rightmost column of Table II assumes this branching ratio between channels 5 and 6 , and adjusts the others accordingly. This reduces channel 5 from $39 \%$ of the total yield to $26 \%$. Because the translational energy distributions in Fig. $6 \mathrm{a}$ and $5 \mathrm{~b}$ are so strikingly similar, it is likely that any overestimation of the channel 5 branching ratio is actually a two-body event from channel 2 that is detected with a third fragment. Thus, that which was subtracted from channel 5 is added to the channel 2 yield, bringing it to $55 \%$. We believe that this procedure yields a more accurate representation of the true photofragment yield.

\begin{tabular}{lrrr}
\hline \multicolumn{3}{c}{ Table II. Product branching fractions at $\mathbf{2 4 8} \mathbf{~ n m}$ and $\mathbf{1 9 3} \mathbf{~ n m}$} \\
\hline Channel & $248 \mathrm{~nm}(\%)$ & $193 \mathrm{~nm}(\%)$ & $\begin{array}{l}193 \mathrm{~nm}(\%) \text { after } \\
\text { correcting channel } \\
\end{array}$ \\
& & & 5 using RRKM \\
\hline (1) $\mathrm{S}+t$-BuS & $90.0 \pm 0.5$ & $7 \pm 1$ & $7 \pm 1$ \\
(2) $\mathrm{S}_{2}+t$-Bu & $10.0 \pm 0.5$ & $42 \pm 5$ & $55 \pm 5$ \\
(5) $\mathrm{S}_{2}+\mathrm{H}+$ isobutene & 0 & $39 \pm 7$ & $26 \pm 7$ \\
(6) $\mathrm{S}_{2}+\mathrm{CH}+$ propene & 0 & $4 \pm 1$ & $4 \pm 1$ \\
(8) $\mathrm{S}+\mathrm{SH}+$ isobutene & 0 & $8 \pm 1$ & $8 \pm 1$ \\
\hline
\end{tabular}

\section{Discussion}

The primary goals of this study are to elucidate the primary photochemistry of the $t$-BuSS and to determine whether photodissociation proceeds via dissociation on an electronically excited state or by decay to the ground electronic state followed by statistical dissociation. Any channel that results in electronically excited products clearly does not proceed by the latter mechanism. The contribution from ground state dissociation can be assessed with reference to Figure 8, which shows a potential energy diagram for the competing two-and three-body dissociation channels of $t$-BuSS on its ground electronic state. Fig. 8 not only shows the asymptotic energetics but also 
the barriers encountered en route to dissociation. Thus, in some cases, radicals produced via two-body dissociation can be formed with enough energy to further dissociate, leading to threebody dissociation products. We can calculate dissociation rate constants for two- and three-body dissociation using RRKM theory, ${ }^{36}$ the methodology and results of which are presented in the supplementary material. With these considerations in mind, we explore in more detail the dynamics and products of the $248 \mathrm{~nm}$ and $193 \mathrm{~nm}$ photodissociation of the $t$-BuSS radical.

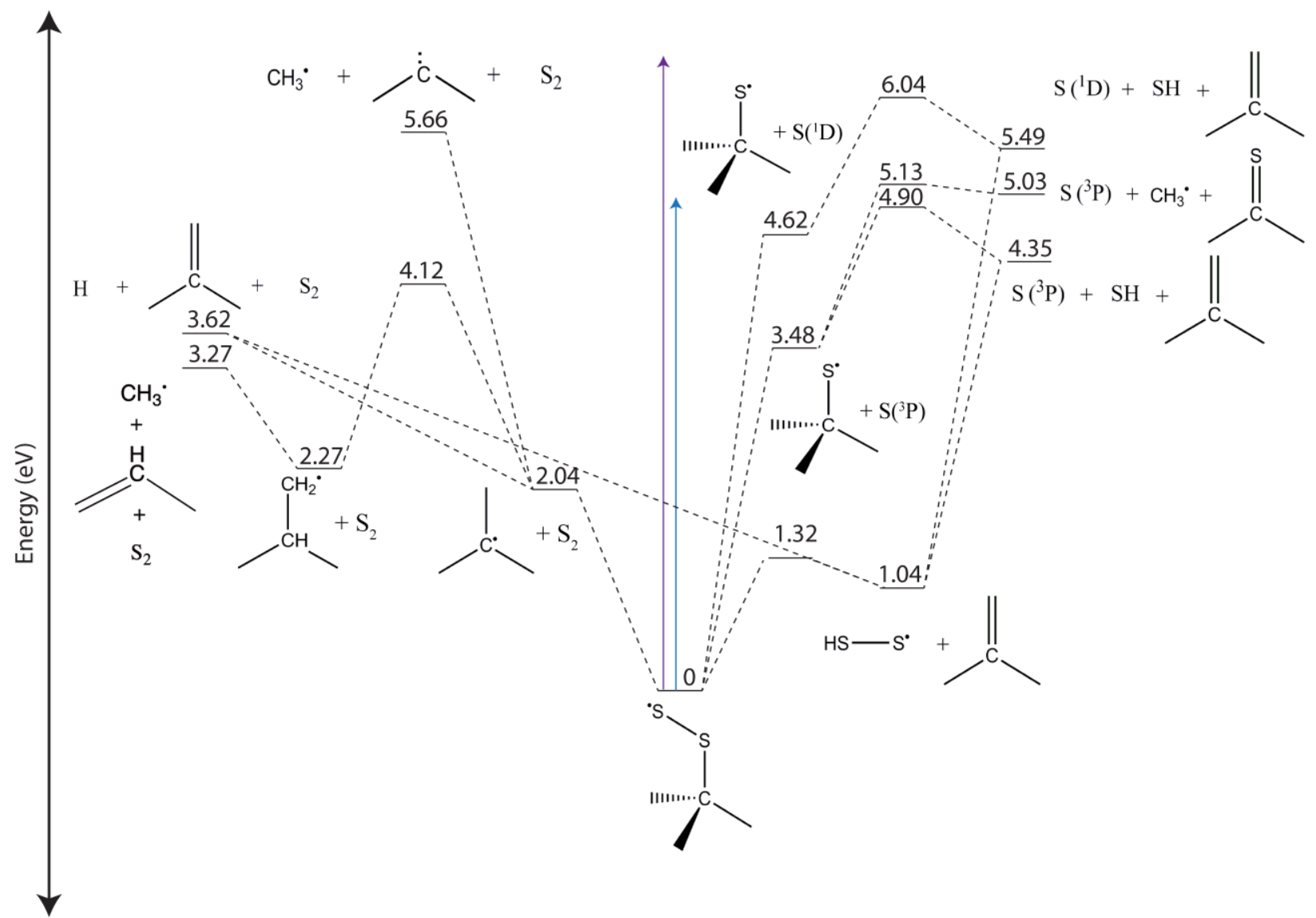

Figure 8. Potential energy diagram for dissociation of $t$-BuSS in which equilibrium geometry energies have been calculated from experimental heats of formation, while transition state energies and geometries were determined at the DFT//B3LYP/aug-cc-pVDZ level of theory or from the literature. ${ }^{40}$ The blue and purple arrows indicate the energies of $248 \mathrm{~nm}$ and $193 \mathrm{~nm}$ photons respectively. 


\section{a. S Loss Dynamics}

As can be seen in Figure 8, at both dissociation energies (5.00 eV and $6.42 \mathrm{eV})$, multiple electronic states of the $\mathrm{S}$ photofragment are energetically accessible. Additionally, the $t$-BuS product can be formed with sufficient internal energy to undergo secondary dissociation to the observed three-body dissociation products $\mathrm{S}+\mathrm{SH}+$ isobutene (channel 8). To determine the electronic states of the $\mathrm{S}$ atom products and the dynamics of their formation and assess the possibility of secondary dissociation of the $t$-BuS radical, we examine the translational energy distributions and angular distributions of these photofragments.

At $248 \mathrm{~nm}, \mathrm{~S}$ loss is the dominant channel, accounting for $90 \%$ of the dissociation products. The translational energy distribution for the $\mathrm{S}$ loss channel in Figure 4 shows a sharp peak at $1.1 \mathrm{eV}$ and cuts off by $1.8 \mathrm{eV}$, close to $E_{\mathrm{T}, \mathrm{MAX}}$ for formation of the ground state products $\mathrm{S}\left({ }^{3} \mathrm{P}\right)+t$-BuS . This is consistent with rapid dissociation on an electronically excited state that is repulsive with respect to $\mathrm{S}$ loss. A repulsive dissociation mechanism is also consistent with the anisotropic distribution of the photofragments. We also note that based on the RRKM branching ratios in Table S4, production of channel 1 by statistical dissociation on the ground state is predicted to be negligible.

At $193 \mathrm{~nm}$, two-body dissociation involving S loss is no longer the dominant product channel, and accounts for only $7 \%$ of the total dissociation products. Inspection of the translational energy distribution of the $\mathrm{S}+t$-BuS product mass channel in Figure 4b shows two distinct peaks: one around $0.1 \mathrm{eV}$ that extends to $0.5 \mathrm{eV}$ and the other around $1.5 \mathrm{eV}$, with intensity out to about 2.5 $\mathrm{eV}$. The lower translational energy peak in the distribution is consistent with the formation of $\mathrm{S}\left({ }^{1} \mathrm{~S}\right)$ on an electronically excited repulsive surface, as the drop-off in intensity of this peak coincides with $E_{\mathrm{T}, \mathrm{MAX}}$ for these products. Additionally, the red trace in Fig. 4b tails off more intensely than the black which is likely an effect of the two detachment wavelengths used. By including internal energy imparted to $t$-BuSS for experiments performed at $532 \mathrm{~nm}$, the full tail of the black trace is accounted for and attributed to $S\left({ }^{1} S\right)$ production. Although the $S\left({ }^{1} \mathrm{D}\right)$ and $\mathrm{S}\left({ }^{3} \mathrm{P}\right)$ electronic states are also energetically accessible in this energy range, the $t$-BuS counterfragment would have internal energy exceeding $1.5 \mathrm{eV}$ and would be unstable with 
respect to secondary fragmentation, in which case we would observe a three-body event rather than a two-body event. Thus, this peak is assigned to the formation of $S\left({ }^{1} S\right)+t$-BuS products on an electronically excited surface.

The feature at higher translational energy lies close to $E_{\mathrm{T}, \mathrm{MAX}}$ for the formation of $\mathrm{S}\left({ }^{1} \mathrm{D}\right)$ and extends nearly to $E_{\mathrm{T}, \mathrm{MAX}}$ for $\mathrm{S}\left({ }^{3} \mathrm{P}\right)$ production. This peak persists to $2.5 \mathrm{eV}$, with some intensity observed all the way to $3.0 \mathrm{eV}$. $E_{\mathrm{T}, \mathrm{MAX}}$ for formation of $\mathrm{S}\left({ }^{1} \mathrm{D}\right)$ is $1.80 \mathrm{eV}$, but some intensity can be seen in Fig. 4b beyond this energy for both photodetachment wavelengths that we attribute to formation of $\mathrm{S}\left({ }^{3} \mathrm{P}\right)$. At translational energies below $\sim 1.5 \mathrm{eV}, t$-BuS fragments resulting from loss of $\mathrm{S}\left({ }^{3} \mathrm{P}\right)$ would have sufficient internal energy to undergo secondary fragmentation, and therefore may contribute to the formation of channel 8. Intensity in the two-body translational energy distribution between 0.5 and $1.5 \mathrm{eV}$ is therefore likely due to the formation of $\mathrm{S}\left({ }^{1} \mathrm{D}\right)$. It thus appears that the higher energy peak in Fig. $4 \mathrm{~b}$ can be mainly attributed to $S\left({ }^{1} \mathrm{D}\right)$ with evidence for small amounts of $\mathrm{S}\left({ }^{3} \mathrm{P}\right)$ formation.

\section{b. S2 Loss Dynamics}

As indicated in Table I, for dissociation of $t$-BuSS to form channel $2\left(\mathrm{~S}_{2}+t\right.$-Bu$)$, multiple electronic states of the $S_{2}$ photofragments are energetically accessible at both dissociation energies. Additionally, the $t$-Bu photofragment may be formed with enough internal energy to undergo secondary dissociation, forming $\mathrm{H}+$ isobutene or $\mathrm{CH}_{3}+$ propene, although three-body signal is seen only at $193 \mathrm{~nm}$. In the following sections, the translational energy and angular distributions and product branching ratios will be examined to gain insight to the products and dynamics of dissociation processes involving $\mathrm{S}_{2}$ loss from $t$-BuSS.

\section{i. Two-body Dynamics: $S_{2}+t$-Bu}

The translational energy distributions for $\mathrm{S}_{2}+t$-Bu are shown in Figure 5. The distributions at the two excitation wavelengths appear similar, with both showing a peak between 0.0 and 1.0 $\mathrm{eV}$, and extending beyond $2.0 \mathrm{eV}$. At the peak of the distribution for dissociation at $248 \mathrm{~nm}, t$-Bu fragments resulting from loss of $S_{2}\left(X^{3} \Sigma_{g}^{-}\right.$) (channel 2A) would have up to $2.6 \mathrm{eV}$ of internal 
energy, assuming no vibrational excitation of the $\mathrm{S}_{2}$ fragment, which would allow the $t$ - $\mathrm{Bu}$ fragment to undergo secondary fragmentation at a considerable rate and therefore be observed as three-fragment dissociation. Since no three-fragment dissociation is observed at $248 \mathrm{~nm}$, formation of product channel $2 \mathrm{~A}$ is unlikely, and the mass channel is therefore assigned to formation of $t$ - $\mathrm{Bu}+\mathrm{S}_{2}$ in the $a^{1} \Delta_{g}$ and/or $b^{1} \Sigma_{g}^{+}$electronic state (channels $2 \mathrm{~B}$ and/or 2C).

The translational energy distribution for $\mathrm{S}_{2}$ loss at $193 \mathrm{~nm}$ (Fig. 5b) has its greatest intensity from 0.5-1.0 eV. Hence, the $t$-Bu fragment resulting from loss $\mathrm{S}_{2}$ in one of its three lowest lying electronic states (channels 2A-2C) would have more than $2.5 \mathrm{eV}$ of internal energy, assuming negligible excitation of the $\mathrm{S}_{2}$ fragment. The energetics and RRKM rate calculations for $t$-Bu dissociation (Table S4) suggest that this situation would result in a three-body event. Therefore, the main peak in the two-body distribution must be due to the production of $S_{2}$ in higher electronic states via channels $2 \mathrm{D}-2 \mathrm{G}$.

We can compare the two-body $\mathrm{S}$ and $\mathrm{S}_{2}$ loss results to those observed in a smaller alkyl perthiyl radical, $\mathrm{CH}_{3} \mathrm{SS}^{8}$ At $248 \mathrm{~nm}, \mathrm{~S}$ loss was found to be the dominant product channel, resulting from rapid excited state dissociation to form ground state $\mathrm{S}$ atoms. For dissociation at $193 \mathrm{~nm}, \mathrm{~S}$ loss remained the dominant channel, although it accounted for a smaller fraction of the products $\left(\mathrm{S}_{2}\right.$ loss being the other channel) than at $248 \mathrm{~nm}$. For $\mathrm{S}_{2}$ loss, the translational energy distribution in the $\mathrm{CH}_{3} \mathrm{SS}$ radical was found to be bimodal due to the formation of multiple electronic states of the $\mathrm{S}_{2}$ photofragment, and extended out to $E_{\mathrm{T}, \mathrm{MAX}}$ for the formation $\mathrm{S}_{2}\left(a^{1} \Delta_{g}\right)+\mathrm{CH}_{3}$ products (equivalent to channel 2B). ${ }^{8}$ This contrasts with the observed translational energy distribution for $t$-BuSS, in which no intensity is observed above $2.5 \mathrm{eV}$, although multiple electronic states of the $\mathrm{S}_{2}$ are also likely formed. The lack of intensity observed at high translational energies in this work suggests that the $\mathrm{S}_{2}+t$-Bu photofragments formed in low electronic states have sufficient energy to dissociate further and are therefore observed as three-body products.

\section{ii. Three-body Dynamics: Secondary Dissociation of $t$-Bu}

At $193 \mathrm{~nm}$, we observe considerable three-body photodissociation. As discussed in the previous section, loss of $\mathrm{S}_{2}$ in the $X^{3} \Sigma_{g}^{-}, a^{1} \Delta_{g}$ or $b^{1} \Sigma_{g}^{+}$electronic states (channels $2 \mathrm{~A}, 2 \mathrm{~B}$, or $2 \mathrm{C}$, 
respectively) can lead to the formation of the $t$-Bu radical that has enough internal energy to further dissociate yielding three-body products. Vibrationally excited $t$-Bu radicals can dissociate via two pathways: direct loss of an $\mathrm{H}$ atom in a barrierless process (channel 5), or isomerization to the iso-butyl radical over a barrier of $2.08 \mathrm{eV}^{40}$ followed by $\mathrm{CH}_{3}$ loss (channel 6). Direct $\mathrm{H}$ atom loss from $t$-Bu requires an energy of $1.58 \mathrm{eV}$ (Figure 8), substantially less than is needed for isomerization to iso-butyl. Indeed, $\mathrm{S}_{2}+\mathrm{H}+$ isobutene is found to be the dominant three-body channel from the calculation of RRKM rate constants (discussed in the supplementary material). The RRKM rate constants predict the dissociation of $t$-Bu to $\mathrm{H}+\mathrm{C}_{4} \mathrm{H}_{8}$ to be on the order of $10^{9}$ $\mathrm{s}^{-1}$, while the production of channel 6 is an order of magnitude less $\left(10^{8} \mathrm{~s}^{-1}\right)$. In both instances, one would expect a small translational energy release (close to $0 \mathrm{eV}$ ) associated with the dissociation of $t$ - $\mathrm{Bu}$, as is observed in Fig. $6 \mathrm{~b}$ for channel 6.

A question of interest is how the $t$-BuSS initially dissociates into $\mathrm{S}_{2}+t$-Bu that then secondarily falls apart to yield channels 5 and 6. An argument in favor of statistical dissociation of $t$-BuSS on its ground state followed by $t$-Bu dissociation is that at $193 \mathrm{~nm}$, channel 2 is the fastest predicted by the RRKM calculations and thus, could reasonably lead to channels 5 and 6 . Based on these calculations, channel 4 should also form, although to a lesser extent, and can fall apart further to channel 5 or channel 8. Additionally, an excited state dissociation of $t$-BuSS leading ultimately to channels 5 and 6 should yield an anisotropic angular distribution for these three-body channels while those observed are isotropic. Perhaps the most convincing argument can be made by referring again to $\mathrm{CH}_{3} \mathrm{SS}$, in which no three-body dissociation was observed. ${ }^{8}$ At $193 \mathrm{~nm}$, the $\mathrm{CH}_{3}+\mathrm{S}_{2}$ translational energy distribution is bimodal, and the authors attributed the high translational energy release peak to formation of electronically excited $S_{2}$ through an excited state mechanism. This is not observed in this work and therefore, suggests that low-lying $\mathrm{S}_{2}$ states $+t$-Bu are formed in a manner such that $t$-Bu has sufficient internal energy to fall apart further. Therefore, a ground state mechanism yielding $t$ - $\mathrm{Bu}$ is most consistent with the available evidence.

Three-body dissociation mechanisms can be classified based on the time interval in which the bonds break. Concerted mechanisms refer to dissociation events in which the breaking of the two bonds occurs in the same kinetic event, whereas in sequential mechanisms the bond cleavages 
are considered as two distinct events. ${ }^{41}$ Concerted processes can be further split depending on whether the bonds are broken simultaneously, or within a rotational period of the molecule, known as synchronous concerted and asynchronous concerted respectively. The Dalitz plot in Figure 7 shows that for the $\mathrm{S}_{2}+\mathrm{CH}_{3}+$ isobutene channel, the $\mathrm{S}_{2}$ fragments are formed with a large fraction of the translational energy, manifesting as an intense stripe at the top of the blue arrow (highlighted by the orange, dashed line). This is most consistent with a sequential mechanism because a relatively constant fraction of translational energy is imparted to the $S_{2}$ fragment. For the dissociation of $t$-Bu to form $\mathrm{H}+$ isobutene, we refer to the RRKM calculations that indicate a dissociation timescale of $\sim 400 \mathrm{ps}$, which is slower than the rotational period of ground state $t$ - $\mathrm{Bu}(\sim 170 \mathrm{ps})$. Therefore, the formation of channel 5 may also be classified a sequential process.

\section{c. Three-body Dissociation to S + SH + Isobutene}

The final product channel observed at $193 \mathrm{~nm}$ corresponds to the formation of $\mathrm{S}+\mathrm{SH}+$ isobutene. As can be seen in Figure 8, there are two possible pathways to form the $\mathrm{S}+\mathrm{SH}+$ isobutene products: $\mathrm{S}$ loss to form $\mathrm{S}+t$ - $\mathrm{BuS}$ followed by secondary dissociation of $t$ - $\mathrm{BuS}$, or formation of $\mathrm{HS}_{2}+$ isobutene followed by secondary dissociation of $\mathrm{HS}_{2}$.

The translational distribution for the $\mathrm{S}+\mathrm{SH}+$ isobutene channel in Fig. 6c shows a main peak around $1.2 \mathrm{eV}$ extending to $\sim 2.0 \mathrm{eV}$, attributed to the formation of $\mathrm{S}$ atoms in the ${ }^{3} \mathrm{P}$ electronic state. A smaller shoulder can be seen below $\sim 0.9 \mathrm{eV}$ which could be from $\mathrm{S}\left({ }^{1} \mathrm{D}\right)+\mathrm{SH}+$ isobutene. Both regions of the distribution peak well away from $0 \mathrm{eV}$, with intensity close to $E_{\mathrm{T}, \mathrm{MAX}}$ for the product channel and show anisotropic distributions of the photofragments.

As discussed in Section 4a, one pathway to these three-body dissociation products involves ground state dissociation of the $t$-BuS fragment after $\mathrm{S}$ loss on an excited state. However, the RRKM rate constant for secondary dissociation of the $t$-BuS radical (Table S4) suggests that the timescale for this ground state dissociation process is much slower than the rotational period. This dissociation mechanism would therefore be expected to lead to an isotropic distribution of photofragments, in contrast to the observed anisotropic distribution. Additionally, RRKM 
calculations suggest that the dominant channel for fragmentation of $t$-BuS would be loss of a methyl radical (to form channel 9) of which, we see little-to-no evidence.

An alternative pathway involves the loss of internally excited $\mathrm{HS}_{2}$ from $t$-BuSS, which is then able to undergo rapid secondary dissociation to form $\mathrm{S}+\mathrm{SH}$. A four-center transition state leading to the loss of vibrationally excited $\mathrm{HS}_{2}$ could be a possible mechanism for this pathway, or $\mathrm{HS}_{2}$ could be produced in an electronically excited state that can then rapidly dissociate into $\mathrm{S}\left({ }^{1} \mathrm{D}\right.$ or $\left.{ }^{3} \mathrm{P}\right)+\mathrm{SH}$ products. Such a mechanism would be expected to impart considerable translational energy into the $\mathrm{S}+\mathrm{SH}$ fragments, consistent with the translational energy distribution for this channel, shown in Figure 6c.

Inspection of the Dalitz plot for $\mathrm{S}\left({ }^{3} \mathrm{P}\right)+\mathrm{SH}+$ isobutene products, integrated between $1.0 \mathrm{eV}$ and $3.0 \mathrm{eV}$, shown in Figure 7c, lends confidence to this mechanism. Here, the region of the Dalitz plot with the most intensity is at the base of the blue arrow, where the energy fraction in the isobutene fragments is very small, and the energy fraction in the S and SH fragments is large, equal and opposite.

The Dalitz plot for formation of the low translational energy shoulder, integrated between 0.0 and $1.0 \mathrm{eV}$ is also consistent with the formation of $\mathrm{S}\left({ }^{1} \mathrm{D}\right)+\mathrm{SH}+$ isobutene products via this mechanism. However, in this case the $\mathrm{S}\left({ }^{1} \mathrm{D}\right)+\mathrm{SH}$ products are higher in energy, and therefore dissociation to these products results in a much smaller translational energy release. Therefore, the Dalitz plot shows maximum intensity in the isobutene fragment (blue arrow), with a much smaller energy fraction found in the $\mathrm{S}\left({ }^{1} \mathrm{D}\right)+\mathrm{SH}$ fragments.

Finally, for production of either $\mathrm{S}^{3} \mathrm{P}$ or ${ }^{1} \mathrm{D}$, the overall mechanism is most appropriately classified as asynchronous concerted, because the secondary dissociation of $\mathrm{HS}_{2}$ is rapid and occurs within its rotational period, a conclusion supported by the anisotropic distribution of the photofragments. 


\section{Conclusions}

We have studied the photodissociation dynamics of the tert-butyl perthiyl radical at $248 \mathrm{~nm}$ and $193 \mathrm{~nm}$ by means of fast beam coincidence translational spectroscopy. S( $\left.{ }^{3} \mathrm{P}\right)$ atom loss was identified as the dominant channel (90\%) for dissociation at $248 \mathrm{~nm}$, with a minor channel forming $\mathrm{S}_{2}+t$-Bu fragments. Translational energy distributions for both processes were found to be consistent with excited state dissociation processes, with $\mathrm{S}$ loss occurring on a repulsive surface. At $193 \mathrm{~nm}$, the photodissociation dynamics are somewhat more complicated, with both two-body and three-body dissociation processes observed. Two-body $\mathrm{S}$ and $\mathrm{S}_{2}$ loss channels were seen, similar to $248 \mathrm{~nm}$ dissociation, however $\mathrm{S}_{2}$ loss becomes the dominant channel. The translational energy distributions for both product channels suggest that $\mathrm{S}$ and $\mathrm{S}_{2}$ loss occur on electronically excited states and result in the formation of multiple electronic states of the $S$ and $\mathrm{S}_{2}$ products.

Three-body dissociation was also observed at $193 \mathrm{~nm}$. The major pathway to three-body dissociation products was found to be a sequential dissociation process in which $\mathrm{S}_{2} \operatorname{loss}$ from $t$ BuSS most likely occurs on the ground state, followed by ground state statistical dissociation of $t$-Bu counterfragments to form $\mathrm{S}_{2}+\mathrm{H}+$ isobutene and $\mathrm{S}_{2}+\mathrm{CH}_{3}+$ propene products. Further three-body dissociation products, $\mathrm{S}+\mathrm{SH}+$ isobutene, were proposed to form in an asynchronous concerted dissociation process via fragmentation of the initially excited $t$-BuSS to produce isobutene + internally excited $\mathrm{HS}_{2}$, which rapidly dissociates into $\mathrm{SH}+\mathrm{S}$.

\section{Supplementary Material}

See supplementary material for simulations, electronic structure calculations, the channel 5 Dalitz plot, and RRKM calculations.

\section{Acknowledgements}

This research was supported by the Director, Office of Basic Energy Science, Chemical Sciences Division of the U.S. Department of Energy under Contract No. DE-AC02-05CH11231 and NIH S10OD023532.

The data that supports the findings of this study are available within the article [and its supplementary material]. 


\section{References}

1. W. J. Wedemeyer, E. Welker, M. Narayan and H. A. Scheraga, Biochemistry 39 (15), 4207-4216 (2000).

2. $\quad$ D. Fass and C. Thorpe, Chem. Rev. 118 (3), 1169-1198 (2017).

3. $\quad$ M. Akiba and A. Hashim, Prog. Polym. Sci. 22 (3), 475-521 (1997).

4. T. Ida, T. Sawa, H. Ihara, Y. Tsuchiya, Y. Watanabe, Y. Kumagai, M. Suematsu, H. Motohashi, S. Fujii and T. Matsunaga, PNAS 111 (21), 7606-7611 (2014).

5. C. L. Bianco, T. A. Chavez, V. Sosa, S. S. Simran, Q. N. N. Nguyen, D. J. Tantillo, A. S. Ichimura, J. P. Toscano and J. M. Fukuto, Free Radical Biol. Med. 101, 20-31 (2016).

6. J.-P. R. Chauvin, M. Griesser and D. A. Pratt, J. Am. Chem. Soc. 139 (18), 6484-6493 (2017).

7. S. Moran and G. B. Ellison, J. Phys. Chem. 92 (7), 1794-1803 (1988).

8. A. W. Harrison, M. Ryazanov, E. N. Sullivan and D. M. Neumark, J. Chem. Phys. 145 (2), 024305 (2016).

9. N. C. Cole-Filipiak, M. Shapero, C. Haibach-Morris and D. M. Neumark, J. Phys. Chem. A 120 (27), 4818-4826 (2016).

10. J. E. Bennett and G. Brunton, J. Chem. Soc., Chem. Commun. (2), 62-63 (1979).

11. T. Burkey, J. Hawari, F. Lossing, J. Lusztyk, R. Sutcliffe and D. Griller, J. Org. Chem. 50 (24), 4966-4967 (1985).

12. J.-P. R. Chauvin, E. A. Haidasz, M. Griesser and D. A. Pratt, Chemi. Sci. 7 (10), 63476356 (2016).

13. A. Gao, H. Du, A. Li and H. Pei, J. Mol. Model. 19 (6), 2443-2449 (2013).

14. M. X. Song, Z. X. Zhao, W. Zhang, F. Q. Bai, H. X. Zhang and C. C. Sun, Int. J. Quantum Chem 112 (6), 1537-1546 (2012).

15. J. Zádor, C. A. Taatjes and R. X. Fernandes, Prog. Energy Combust. Sci. 37 (4), 371-421 (2011).

16. B. Nichols, E. N. Sullivan, M. Ryazanov, C. M. Hong and D. M. Neumark, J. Chem. Phys. 147 (13), 134304 (2017).

17. E. N. Sullivan, B. Nichols and D. M. Neumark, J. Chem. Phys. 148 (4), 044309 (2018).

18. J. Hawari, D. Griller and F. Lossing, J. Am. Chem. Soc. 108 (12), 3273-3275 (1986).

19. B. Ruscic and J. Berkowitz, J. Chem. Phys. 98 (4), 2568-2579 (1993).

20. M. W. Chase, NIST-JANAF Thermochemical Tables, $4^{\text {th }}$ ed. (NIST, Gaithersberg, MD, 1998) 1-1951.

21. R. E. Continetti, D. R. Cyr, D. L. Osborn, D. J. Leahy and D. M. Neumark, J. Chem. Phys. 99 (4), 2616-2631 (1993).

22. A. A. Hoops, J. R. Gascooke, A. E. Faulhaber, K. E. Kautzman and D. M. Neumark, Chem. Phys. Lett. 374 (3), 235-242 (2003).

23. M. Ryazanov, A. W. Harrison, G. Wang, P. E. Crider and D. M. Neumark, J. Chem. Phys. 140 (23), 234304 (2014).

24. D. Irimia, D. Dobrikov, R. Kortekaas, H. Voet, D. A. van den Ende, W. A. Groen and M. H. Janssen, Rev. Sci. Instrum. 80 (11), 113303 (2009).

25. E. Garand, T. I. Yacovitch and D. M. Neumark, J. Chem. Phys. 130 (6), 064304 (2009).

26. J. M. B. Bakker, J. Phys. E. 6 (8), 785 (1973).

27. J. M. B. Bakker, J. Phys. E. 7 (5), 364 (1974). 
28. V. Dribinski, A. Ossadtchi, V. A. Mandelshtam and H. Reisler, Rev. Sci. Instrum. 73 (7), 2634-2642 (2002).

29. O. Jagutzki, A. Cerezo, A. Czasch, R. Dörner, M. Hattas, M. Huang, V. Mergel, U. Spillmann, K. Ullmann-Pfleger, T. Weber, H. Schmidt-Böcking and G. D. W. Smith, IEEE Trans. Nucl. Sci. 49 (5), 2477-2483 (2002).

30. A. W. Harrison, J. S. Lim, M. Ryazanov, G. Wang, S. Gao and D. M. Neumark, J. Phys. Chem. A 145, 024305 (2013).

31. A. W. Harrison, J. S. Lim, M. Ryazanov, G. Wang, S. Gao and D. M. Neumark, The Journal of Physical Chemistry A 117 (46), 11970-11978 (2013).

32. V. A. Mozhayskiy and A. I. Krylov, ezSpectrum3.0, iOpenShell Center for Computational Studies of Electronic Structure and Spectroscopy of Open Shell and Electronically Excited Species, Los Angeles, http://iopenshell.usc.edu/downloads (2009).

33. Gaussian 09, Revision D.01, M. J. Frisch, G. W. Trucks, H. B. Schlegel, G. E. Scuseria, M. A. Robb, J. R. Cheeseman, G. Scalmani, V. Barone, B. Mennucci, G. A. Petersson, H. Nakatsuji, M. Caricato, X. Li, H. P. Hratchian, A. F. Izmaylov, J. Bloino, G. Zheng, J. L. Sonnenberg, M. Hada, M. Ehara, K. Toyota, R. Fukuda, J. Hasegawa, M. Ishida, T. Nakajima, Y. Honda, O. Kitao, H. Nakai, T. Vreven, J. A. Montgomery Jr., J. E. Peralta, F. Ogliaro, M. J. Bearpark, J. Heyd, E. N. Brothers, K. N. Kudin, V. N. Staroverov, R. Kobayashi, J. Normand, K. Raghavachari, A. P. Rendell, J. C. Burant, S. S. Iyengar, J. Tomasi, M. Cossi, N. Rega, N. J. Millam, M. Klene, J. E. Knox, J. B. Cross, V. Bakken, C. Adamo, J. Jaramillo, R. Gomperts, R. E. Stratmann, O. Yazyev, A. J. Austin, R. Cammi, C. Pomelli, J. W. Ochterski, R. L. Martin, K. Morokuma, V. G. Zakrzewski, G. A. Voth, P. Salvador, J. J. Dannenberg, S. Dapprich, A. D. Daniels, Ö. Farkas, J. B. Foresman, J. V. Ortiz, J. Cioslowski and D. J. Fox, Gaussian, Inc., Wallingford, CT, USA, 2013.

34. D. R. Cyr, D. J. Leahy, D. L. Osborn, R. E. Continetti and D. M. Neumark, J. Chem. Phys. 99 (11), 8751-8764 (1993).

35. M. Tashiro, Chem. Phys. Lett. 453 (4-6), 145-149 (2008).

36. R. A. Marcus and O. Rice, J. Phys. Chem. 55 (6), 894-908 (1951).

37. R. H. Dalitz, Philos. Mag. 44 (357), 1068-1080 (1953).

38. D. Babikov, E. A. Gislason, M. Sizun, F. Aguillon, V. Sidis, M. Barat, J. C. Brenot, J. A. Fayeton and Y. J. Picard, J. Chem. Phys. 116 (12), 4871-4876 (2002).

39. D. E. Szpunar, A. E. Faulhaber, K. E. Kautzman, P. E. Crider II and D. M. Neumark, J. Chem. Phys. 126 (2007).

40. B. Noller, R. Maksimenka, I. Fischer, M. Armone, B. Engels, C. Alcaraz, L. Poisson and J.-M. Mestdagh, J. Phys. Chem. A 111 (10), 1771-1779 (2007).

41. C. Maul and K.-H. Gericke, Int. Rev. Phys. Chem. 16 (1), 1-79 (1997). 